\title{
CONVENIÊNCIA DE SERVIÇOS: APROPRIAÇÃO E ADAPTAÇÃO DE UMA ESCALA DE MEDIDA
}

\author{
SERVICE CONVENIENCE: APPROPRIATION AND ADAPTATION OF A \\ MEASUREMENT SCALE
}

CONVENIENCIA DE SERVICIOS: APROPIACIÓN Y ADAPTACIÓN DE UNA ESCALA DE MEDIDA

\section{RESUMO}

O trabalho busca adequar a Escala de Conveniência de Serviços - Servcon (SEIDERS, VOSS, GODFREY, 2007) para uso no contexto brasileiro, mais especificamente no varejo de livre localização das cidades de porte médio. Assume, a priori, que as cinco dimensões utilizadas na escala original não são suficientes para representar a conveniência de serviços na realidade brasileira, por ignorar importantes fatores de (in)conveniência, como aqueles decorrentes da insegurança, da informalidade e da desorganização urbana. Após a etapa exploratória, feita por meio de grupo focal, foram colhidas duas amostras de respondentes, uma formada por 268 universitários e outra por 206 consumidores não acadêmicos. O trabalho acrescenta duas novas dimensões à Servcon original, cuja versão ampliada apresenta índices razoáveis de ajustamento geral, de confiabilidade e de variância extraída em todos os construtos. Os resultados evidenciam que fatores relacionados à segurança, à mobilidade e ao regramento da informalidade precisam ser incorporados ao estudo da conveniência, para adequá-la ao contexto brasileiro.

PALAVRAS-ChAVE Conveniência, serviços, varejo, escala, medida.

\author{
Edar da Silva Añaña edaranana@gmail.com \\ Professor da Faculdade de Administração e de Turismo, Universidade Federal de Pelotas - Pelotas - RS, Brasil
}

Renata Gonçalves Santos Silva renata.ssv@gmail.com

Professora da Faculdade de Administração e de Turismo, Universidade Federal de Pelotas - Pelotas - RS, Brasil

Walter Meucci Nique wmnique@ea.ufrgs.br

Professor da Escola de Administração, Universidade Federal do Rio Grande do Sul - Porto Alegre - RS, Brasil

\begin{abstract}
The work seeks to fit the Service Convenience Scale - SERVCON (SEIDERS, VOSS and GODFREY, 2007), to the Brazilian retail reality, in particular to the free-stand location commerce. We Assume that the five dimensions identified in the original work are not broad enough to permit proper evaluation of service convenience in most of the Brazilian cities reality. The research involved a focus group to identify a wider set of variables and two surveys: the first one collected a sample of 268 undergraduate students and the second one surveyed 206 non academic customers. Two new dimensions were identified and added to the five original ones with acceptable levels of fit and validity. Results indicated that lack of security, barriers restricting customers' mobility, and bad regulation of informality interfere in the convenience assessment and therefore must be included in the instrument.

keywords Convenience, services, retail, scale, measurement.

Resumen El trabajo busca adecuar la Escala de Conveniencia de Servicios - Servcon (SEIDERS, VOSS, GODFREY, 2007) para uso en el contexto brasileño, más especificamente en el comercio minorista de libre localización de las ciudades de porte medio. Asume, a priori, que las cinco dimensiones utilizadas en la escala original no son suficientes para representar la conveniencia de servicios en la realidad brasileña, por ignorar importantes factores de (in) conveniencia, como aquellos derivados de la inseguridad, de la informalidad y del desorden urbano. Después de la etapa exploratoria, hecha por medio de grupo focal, fueron recogidos dos muestras de encuestados, una formada por 268 estudiantes universitarios y otra por 206 consumidores no académicos. El trabajo acrecienta dos nuevas dimensiones a la Servcon original que presenta en su versión ampliada índices razonables de ajustamiento general, de confiabilidad y de varianza extraída en todos los constructos. Los resultados evidencian que factores relacionados a la seguridad, a la movilidad e al reglamento de la informalidad han de ser incorporados al estudio de la conveniencia para adaptarla al contexto brasileño.
\end{abstract}

Palabras clave Conveniencia, servicios, comercio minorista, escala, medida. 


\section{INTRODUÇÃO}

Os shopping centers nasceram nos Estados Unidos, nos anos 1950, como espaços de compras, lazer e descanso, focados principalmente nos consumidores em trânsito entre a residência e o trabalho e vice-versa. No início, localizavam-se em regiões de fácil acesso, nos entroncamentos das rodovias que ligavam as grandes metrópoles às pequenas cidades e às comunidades do seu entorno, em áreas amplas e relativamente baratas (SCOTTI, 2007). Ao longo do tempo, conforme as cidades foram crescendo e a sociedade evoluindo, $\mathrm{O}$ modelo foi tomando novas formas e sendo paulatinamente incorporado à paisagem urbana.

O modelo chegou ao Brasil em 1966, com o Shopping Iguatemi, em São Paulo, e dali para cá não parou mais de crescer. Ao contrário dos Estados Unidos, o shopping center brasileiro já nasceu urbano, movido principalmente pela necessidade de segurança. Segundo Scotti (2007), "o shopping center brasileiro é hoje um sucesso, não pelo seu conceito original, mas por oferecer segurança - avis rara em qualquer centro urbano no Brasil". Segundo esse autor, o consumidor brasileiro está sendo "empurrado" para os shoppings, muito mais pela incapacidade do poder público de prover a segurança necessária aos seus cidadãos do que pelas virtudes daquele modelo de organização. Em outras palavras, o que leva os consumidores brasileiros a trocar o comércio tradicional de livre localização pelos shopping centers não é a conveniência, mas sim a insegurança das ruas.

No Brasil moderno, tanto os shopping centers quanto as lojas de livre localização têm a sua conveniência afetada por uma multiplicidade de fatores. Afora a insegurança, que afeta principalmente o comércio de livre localização (SCOTTI, 2007), outros fatores, como o transporte coletivo precário, o comércio informal, a mendicância, o trânsito caótico e tantos outros, também podem estar contribuindo para a percepção de conveniência (ou da falta desta). Em alguns serviços, especialmente nas cidades pequenas e médias, boa parte da população ainda depende do comércio convencional para suprir suas necessidades, seja pela ausência de shopping centers, seja pelo aspecto excludente desse modelo (YAMAWAKI e DUARTE, 2010). O presente trabalho discute a conveniência no varejo de livre localização, aquele em que as lojas se localizam individualmente ou em pequenas galerias ao longo de ruas e avenidas, expostas à inclemência do tempo, à insegurança da aglomeração, à falta de estacionamento e a outros fatores de desconforto.
O estudo da conveniência de serviço começou a aparecer com mais frequência na literatura de marketing a partir do início do século XXI. Baker e outros (2002) incluem o esforço de tempo na formação do valor para o cliente; Meuter e outros (2000) e Collier e Sherrell (2010) incluem a conveniência entre os fatores geradores de satisfação, especialmente nos autosserviços. Mas o construto como um todo somente foi individualizado com o trabalho de Berry, Seiders e Grewal (2002), que propuseram um modelo de conveniência de serviço baseado em cinco dimensões: conveniência de decisão, de acesso, de transação, de benefício e de pós-benefício, o qual não chegou a ser testado empiricamente na época em que foi concebido.

Dois trabalhos recentes buscaram criar e validar escalas para medir o construto proposto por Berry, Seiders e Grewal (2002). O primeiro, de Seiders, Voss e Godfrey (2007), propõe uma escala de mensuração genérica - a Servcon - que, segundo os autores, deveria ser capaz de medir a conveniência em diversos tipos de serviços, incluindo o varejo. E o segundo, de Colwell e outros (2008), propõe uma escala com características mais ou menos semelhantes, porém utilizando como referência o serviço de telefonia móvel, um serviço que, segundo os próprios autores reconhecem, tem características próprias, já que a interação com o consumidor frequentemente se inicia fora do local de prestação dos serviços (em casa, por exemplo), e não na loja, como a maioria dos demais.

Ainda que as duas escalas criadas tenham se mostrado confiáveis e válidas no contexto onde foram testadas, elas refletem uma realidade um tanto distante das cidades brasileiras, em especial das cidades de médio porte, onde as lojas individuais (freestanding shops) ainda resistem, apesar do desconforto e da insegurança. O comércio convencional, localizado ao longo de ruas de acesso público, resgata alguma nostalgia, na medida em que permite a livre expressão e a interação cultural; é um espaço para interagir e transgredir, que reflete uma espécie de antimodernismo ou de resistência à comodidade. Entretanto, a instalação de shopping centers nas cidades médias e grandes está tornando cada vez mais raro esse tipo de organização comercial. Segundo Goss (1993 p. 25), nos Estados Unidos, o comércio de livre localização "já não mais existe [nos grandes centros urbanos], tendo em vista que os poucos espaços abertos que restam funcionam apenas como túneis entre os edifícios, e encontram-se hoje abandonados à população marginal". 
O presente trabalho parte do pressuposto de que tanto a definição do construto quanto os instrumentos de medida que foram desenvolvidos anteriormente necessitam de adequações para que sejam aplicáveis em economias menos desenvolvidas, onde as redes de serviços não têm uniformidade e capilaridade comparáveis às dos países desenvolvidos. Alguns aspectos, como a conveniência de decisão e de acesso, precisam ser mais bem definidos para que reflitam a realidade brasileira, incorporando alguns aspectos que foram ignorados na construção da Servcon original (como o trânsito, a informalidade e a insegurança, por exemplo) e explorando algumas relações existentes entre as diversas facetas da conveniência, "que raramente são consideradas pelos gestores" (SEIDERS, BERRY e GRESHAM, 2000, p. 79).

\section{ESTRUTURA CONCEITUAL}

A conveniência, definida inicialmente como um atributo do produto, capaz de reduzir tempo e esforço de compra, evoluiu com o tempo e passou a ser vista como o custo de tempo e de esforço utilizados para a compra, e não mais como um atributo do produto em si (BROWN, 1990). Baseados em recursos como o tempo, a oportunidade e a energia a que os consumidores renunciam para comprar os bens e os serviços de que necessitam, alguns pesquisadores passaram a ver a falta de conveniência como um preço não monetário a ser suportado pelos consumidores (BERRY, SEIDERS, GREWAL, 2002).

Segundo Colwell e outros (2008), o conceito foi trazido para a literatura de marketing por Copeland, em 1923, que classificou de "bens de conveniência" os produtos distribuídos intensivamente, cuja compra requer o mínimo de esforço físico, mental e de tempo. Mais adiante, a conveniência passou a ser associada ao tempo e ao esforço envolvidos na aquisição de bens e serviços (BROWN, 1990; BROWN E MCENALLY, 1993), como um atributo que reduz o custo não monetário, ou como um componente dos aspectos físicos que compõem o ambiente de loja (DABHOLKAR, THORPE, RENTZ, 1996). Grandezas de difícil contabilização, o tempo e o esforço formam parte dos chamados custos não monetários: o tempo é vendido pelos consumidores no mercado de trabalho e "recomprado" mais adiante, incorporado aos produtos e serviços poupadores de tempo (alimentos prontos ou semiprontos, por exemplo); e o mesmo acontece com a energia e o esforço (físico, mental ou psicológico) requeridos para a obtenção dos serviços, cujos custos não são facilmente contabilizáveis, mas que, nem por isso, deixam de influenciar a percepção de conveniência (SEIDERS, BERRY, GRESHAM, 2000, p. 79).

Consumidores percebem a conveniência de diferentes maneiras, conforme o tipo de serviço que compram ou que usam. As características que mais influenciam a conveniência dos serviços são as consequências, a inseparabilidade, a limitação de suprimento, a intensidade de trabalho requerido e o valor hedônico (BERRY, SEIDERS, GREWAL, 2002). A natureza e o tipo de serviço influenciam a sensibilidade ao tempo, e, dessa forma, alguns tipos de conveniência podem ser mais afetados do que outros.

A economia de tempo e de esforços são os dois aspectos da conveniência mais citados na literatura. Berry, Seiders e Grewal (2002) propõem cinco tipos de conveniência que, segundo eles, correspondem às atividades relacionadas à aquisição e ao uso dos serviços: (1) conveniência de decisão; (2) conveniência de acesso; (3) conveniência de transação; (4) conveniência de benefício; e (5) conveniência pós-benefício.

A conveniência de decisão está relacionada à escolha do serviço a ser utilizado e do seu fornecedor. O consumidor que deseje determinada performance de serviço terá que gastar tempo e esforço para decidir como obtê-lo. Primeiro, terá que decidir entre fazer ele próprio ou adquirir o serviço; e, após, caso decida pela aquisição do serviço, terá que decidir de quem e que tipo específico de serviço comprar (BERRY, SEIDERS, GREWAL, 2002). Como a decisão antecede à escolha, a abundância e a qualidade das informações disponíveis são fundamentais nessa etapa, particularmente quando envolvem serviços complexos ou difíceis de serem avaliados, nos quais o limiar de decisão é mais alto (ZEITHAML, BERRY, PARASURAMAN, 1996).

A conveniência de acesso envolve a rapidez e a facilidade com que os consumidores conseguem chegar ao prestador e se engajar na prestação do serviço, seja pessoalmente, seja por telefone, pela internet ou por outros meios. Por isso, a localização, o horário de funcionamento, a facilidade de estacionamento e as opções de contato remoto figuram entre os principais elementos da conveniência de acesso entre os serviços que requerem a presença dos consumidores. E, assim como as demais formas, a conveniência de acesso é indispensável ao varejo, inclusive ao chamado varejo sem loja, uma vez que, sem acessibilidade, todas 
as demais formas de conveniência são irrelevantes (SEIDERS, BERRY, GRESHAM, 2000).

A conveniência de transação envolve o custo de tempo e de esforço percebido pelo consumidor para completar a transação. É um tipo de conveniência que foca estritamente nas ações que o consumidor é solicitado a realizar para assegurar o direito ao uso do serviço. Como os serviços em geral presumem a existência de troca - normalmente dinheiro por uma promessa de performance - a conveniência de transação envolve basicamente a facilidade com que o consumidor consegue completar a transação na qual se envolveu. Questões como a baixa performance dos checkouts ou o longo tempo de espera em filas de saída, representam custos de oportunidade para os clientes e, por essa razão, são vistos como inconvenientes importantes (BERRY, SEIDERS, GREWAL, 2002).

A conveniência de benefício pode ser entendida como o tempo e o esforço despendidos pelo consumidor para usufruir o benefício central do serviço, como "ser transportado" por um táxi ou por um avião, ou mesmo "falar" ao celular. Dessa forma, é fundamental que outros inconvenientes não interfiram no acesso do consumidor a esse tipo de benefício (BERRY, SEIDERS, GREWAL, 2002). É o caso, por exemplo, dos voos internacionais que precisam ser conectados a trechos domésticos, e cujas conexões são perdidas por falta de agilidade alfandegária nos aeroportos de entrada. Nesse caso, de pouco ou nada adianta ao consumidor que os voos cheguem e partam no horário se ele, por razões alheias à vontade da companhia aérea, não consegue usufruir do benefício central contratado, que é voar até o seu destino.

E a conveniência pós-benefício envolve o esforço e o tempo necessários para reiniciar o contato com o fornecedor após o benefício do serviço. A conveniência pós-benefício pode estar relacionada a uma reclamação, uma solicitação de assistência ou de troca, ou da tentativa de fazer alguma atualização do produto ou complementação do serviço adquirido. Pesquisas anteriores têm demonstrado que a experiência pós-consumo, quando adequadamente gerida, pode influir positivamente na satisfação e na lealdade dos clientes (SANTOS e ROSSI, 2002; SANTOS e FERNANDES, 2008).

Afora as cinco dimensões descritas na literatura revisada, o presente trabalho também investiga as relações entre elas e considera outras variáveis que podem contribuir para a percepção de conveniência ou da sua falta. É o caso, por exemplo, da violência urbana (CHESNAIS, 1999), dos guardadores informais de auto- móveis - os "flanelinhas" (ALCÂNTARA, 2005; FERREIRA, 2006) e dos vendedores ambulantes, cuja atuação é ostensiva de norte a sul do Brasil e cuja presença pode interferir na formação da conveniência. A questão da insegurança, em algumas cidades, é tão evidente que muitos consumidores sentem-se "empurrados" para os shopping centers, mesmo que não tenham predileção por aquele modelo de varejo (SCOTTI, 2007).

\section{MÉTODO}

O trabalho aqui apresentado segue o desenho de pesquisa adotado por Seiders, Voss e Godfrey (2007) para desenvolvimento da Servcon. Dadas as características da pesquisa, o estudo foi realizado em duas etapas, uma exploratória e outra descritiva, conforme será visto a seguir.

\section{Etapa exploratória}

$\mathrm{Na}$ fase exploratória da pesquisa, buscou-se verificar a compreensão e adequação da escala à realidade brasileira, identificar possíveis novas variáveis de interesse e formular as hipóteses de pesquisa. A apropriação da escala envolveu as seguintes atividades: (a) tradução para o português por dois dos autores fluentes em inglês, (b) retrotradução da primeira versão para o inglês por tradutora profissional e, finalmente, (c) comparação da nova versão em inglês com a escala original. A versão retrotraduzida apresentou pequenas diferenças em relação à escala original, e a versão em português foi considerada satisfatória.

A definição dos fatores de conveniência e das possíveis inter-relações entre eles foi feita por meio de grupo de foco, e envolveu 12 pessoas, entre varejistas e outros empresários do setor de serviços, gestores públicos, gerentes, vendedores, taxistas, donas de casa e outros consumidores. O trabalho foi coordenado por um dos autores previamente treinado para tal fim e utilizou um roteiro que incluía, entre outras, as cinco dimensões da escala original. O vídeo da reunião foi transcrito posteriormente para identificação do rol de variáveis de interesse e formulação de novas hipóteses de pesquisa.

\section{Estudo piloto}

Previamente à coleta de dados propriamente dita, o questionário foi respondido por um grupo de 10 es- 
tudantes do Curso de Administração de uma universidade do Rio Grande do Sul, para verificar possíveis dificuldades de interpretação. A coleta dessa amostra piloto foi feita pelos próprios pesquisadores de campo, sob supervisão dos autores, e nenhuma dificuldade maior foi identificada.

\section{Etapa descritiva}

A etapa descritiva foi feita em dois tempos: um primeiro estudo envolvendo uma amostra de 268 estudantes de diferentes carreiras universitárias, e um segundo estudo envolvendo 206 consumidores não acadêmicos. No primeiro estudo, foi empregado um questionário com 64 questões, compreendendo as variáveis da Servcon acrescidas de um elenco de possíveis novas variáveis de interesse, levantadas no grupo de foco. E, no segundo estudo, empregou-se um questionário com 45 questões, incluindo a escala já purificada e as variáveis hipotetizadas como possíveis antecedentes e consequentes do construto. O perfil e outras características da amostra são apresentados mais adiante, junto aos resultados quantitativos.

\section{RESULTADOS DO TRABALHO EXPLORATÓRIO}

Como a pesquisa tinha por objetivo testar e apropriar uma escala desenvolvida em outra realidade econômico-mercadológica, para uso no Brasil, já era possível supor que nem todas as variáveis originais se adequassem ao contexto nacional, e que outras novas pudessem surgir. A retrotradução da escala original foi feita antes da realização do grupo de foco, para que os fatores de conveniência já testados empiricamente pudessem ser utilizados como norteadores da discussão.

As discussões realizadas durante o grupo de foco lançaram alguns insights para a formulação de novas hipóteses de pesquisa, além daquelas baseadas na literatura precedente. Entre outros caminhos, o trabalho exploratório sugere o seguinte:

a) Todos os itens da Servcon (SEIDERS, VOSSA, GODFREY, 2007) constituem-se em fatores de (in)conveniência também no Brasil e, portanto, devem ser mantidos no instrumento de coleta.

b) Além dos itens da escala original, outros fatores também contribuem para a percepção de conve- niência nos serviços em geral, mormente no comércio varejista, seja por facilitarem/dificultarem a decisão ou o acesso aos serviços, seja por tornarem estes mais ou menos benéficos aos consumidores.

c) Algumas questões, como a segurança pública, a complexidade do trânsito, a existência de transporte coletivo e de serviços de tele-entrega, a qualidade dos equipamentos públicos como passarelas, faixas de segurança, sinalização etc., podem contribuir para a conveniência de acesso ou de decisão, ou mesmo formar uma dimensão à parte.

d) A existência de barreiras vivas, como vendedores ambulantes, "guardadores" de carros, cães errantes, pedintes etc., podem influenciar a conveniência de decisão, de acesso ou de pós-benefício, ou mesmo formar uma dimensão à parte.

e) Algumas dimensões da Servcon podem apresentar relação de ancestralidade entre si (SEIDERS, BERRY, GRESHAM, 2000). É o caso, por exemplo, da conveniência de decisão, que pode ser influenciada pela dificuldade de acesso a um prestador de serviços ou pelos benefícios obtidos em eventos anteriores.

\section{HIPÓTESES DE PESQUISA}

Com base no trabalho exploratório e na literatura revisada, é possível identificar algumas relações teóricas entre os construtos. O resultado da primeira etapa da pesquisa sugere, entre outras coisas, que, no contexto brasileiro, a conveniência não se restringe às variáveis propostas por Seiders, Voss e Godfrey (2007). Ainda que a escala original tenha se mostrado válida no contexto onde foi testada, ela não cobre alguns aspectos importantes da conveniência, como o tráfego, a (in) segurança e a informalidade. E, além da incorporação de novos fatores, o resultado do grupo de foco sugere, ainda, a existência de algumas relações de ancestralidade entre as dimensões já identificadas, conforme será visto a seguir. A Figura 1 apresenta um resumo das hipóteses propostas.

O prazer de "ir às compras" refere-se ao afeto positivo que é despertado no consumidor quando este se envolve na busca de produtos de seu interesse (SEIDERS, VOSS, GODFREY, 2007). Consumidores hedonistas consideram a busca e a compra propriamente dita uma experiência recompensadora e praze- 
rosa (ARNOLD e REYNOLDS, 2003) e, devido a isso, não percebem os custos de tempo e de esforço com a mesma intensidade que os demais consumidores.

H1: O prazer demonstrado pelos consumidores nas atividades de compra favorece a percepção de conveniência, em todas as suas dimensões.

O envolvimento reflete o grau de importância que uma determinada categoria de produto tem para o consumidor, fazendo com que este dedique maior tempo à pesquisa dos itens de seu interesse (BEATTY e SMITH, 1987). O conhecimento adquirido durante as sucessivas experiências de compra leva os consumidores a conhecer melhor as alternativas existentes no comércio local e reduz o tempo e o esforço necessários à busca, aumentando, assim, a conveniência de decisão e de benefício (SEIDERS, VOSS, GODFREY, 2007).

$\mathrm{H} 2$ : O envolvimento com os produtos habitualmente vendidos em determinada loja favorece a percepção de conveniência de decisão e de benefício.

O deslocamento até o local de prestação do serviço expõe o consumidor a diversos riscos. No caso do comércio de livre localização, mormente em cidades onde a segurança pública deixa a desejar, além dos riscos inerentes ao deslocamento, existe, ainda, o risco de ser assaltado no interior, na entrada ou na saída da loja. A situação é tão grave, no Brasil de hoje, que muitos consumidores chegam a trocar as lojas de livre localização pelos shopping centers em função da insegurança (SCOTTI, 2007).

H3: A sensação de segurança favorece a conveniência de decisão e influencia positivamente a satisfação, a recompra e a recomendação da loja.

A concentração de pessoas e de veículos em áreas tipicamente comerciais dificulta a mobilidade dos consumidores, mas favorece a simbiose entre as empresas devido à complementariedade e à diluição dos custos fixos das "viagens de compras" (GIJSBRECHTS, CAMPO, NISOL, 2008). Fatores como o trânsito intenso, a desorganização das vias de circulação, a deficiência de equipamentos urbanos (sinalização, túneis, passarelas etc.) e o comércio informal dificultam a circulação e causam transtornos aos consumidores. Entretanto, em cidades menores, como a que foi empregada no presente estudo, a área central concentra o grande comércio e as repartições públicas, e a aglomeração constitui-se num atrativo para os consumidores, que encontram numa mesma área todos os itens da sua lista de compras, reduzindo os custos de transação (MILLER, REARDON, MCCORKLE, 1999).

\begin{abstract}
H4a: A localização da loja em áreas de grande concentração comercial reduz a mobilidade das pessoas. H4b: A localização da loja em áreas de grande concentração comercial influencia positivamente a conveniência de acesso da loja.

H4c: A localização da loja em áreas de grande concentração comercial faz com que os clientes sofram maior interferência dos inconvenientes urbanos.
\end{abstract}

As barreiras físicas e humanas, que surgem nas áreas de comércio informal intenso, dificultam a circulação, especialmente das pessoas mais velhas (GIL, 2011). E o mesmo pode ser dito em relação aos animais abandonados (AMIGOS DE PELOTAS, 2008) e aos "guardadores" de carros (MELLO, 2011), que prejudicam a circulação e causam desconforto entre os consumidores, especialmente entre os mais velhos.

H5: A existência de barreiras vivas prejudica a mobilidade e causa desconforto, especialmente entre as mulheres e os mais velhos.

A dificuldade em deslocar-se de casa até o local de comércio, ou mesmo de circular por entre as lojas (no caso do comércio de livre localização), aumenta o tempo e o esforço do consumidor para a obtenção do serviço. E, como a formação do valor para o cliente envolve recompensas e custos (ZEITHAML, 1988), a dificuldade para locomover-se tende a tornar os serviços menos satisfatórios para os clientes.

H6: A mobilidade influi positivamente na satisfação, na recompra e na recomendação da loja.

Segundo Seiders, Voss e Godfrey (2005), a conveniência interage com a satisfação e influencia positivamente a decisão de recompra e o aumento dos gastos no mesmo fornecedor. Consumidores satisfeitos, por seu turno, são mais propensos a falar positivamente sobre a empresa (ANDERSON, 1998).

H7: A conveniência influencia diretamente a satisfação dos consumidores com os serviços da loja. 
H8: A satisfação influencia positivamente a decisão de recomprar e de recomendar os serviços da loja.

Para Seiders, Voss e Godfrey (2007), as conveniências de decisão e de acesso são salientes antes mesmo de ocorrer a chamada troca de serviço; as conveniências de transação e de benefício tornam-se salientes quando a troca de serviço se inicia; e a conveniência pós-benefício somente ganha importância após a troca de serviço.

H9: A conveniência de decisão é influenciada diretamente pelas conveniências de acesso, de benefício, de transação e de pós-benefício.

\section{Figura 1 - Resumo das hipóteses de pesquisa}

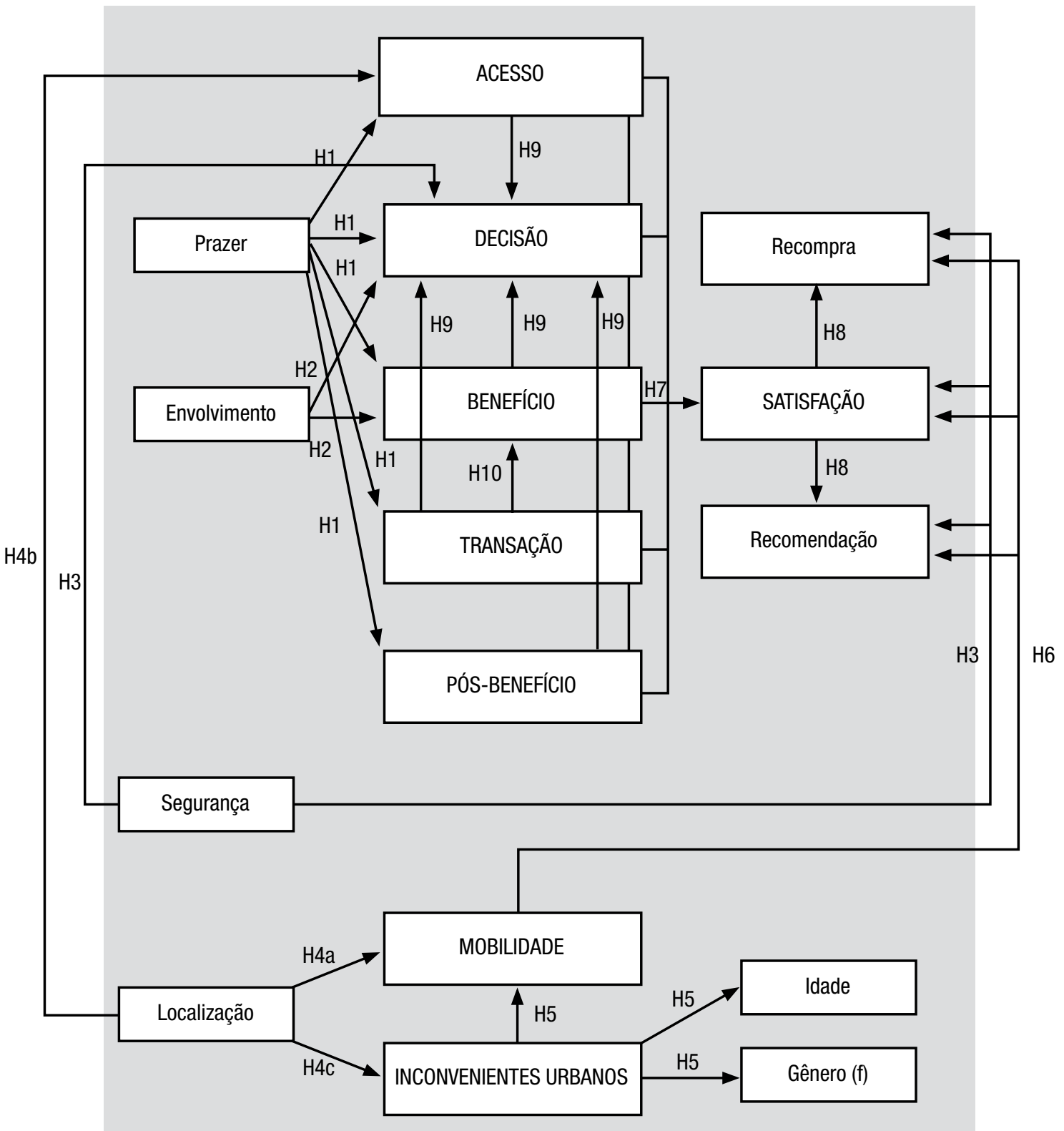


H10: A conveniência de benefício é influenciada pela conveniência de transação.

O próximo item apresenta os resultados dos dois estudos descritivos.

\section{RESULTADOS DO TRABALHO DESCRITIVO}

A seguir, são apresentados os resultados dos dois estudos realizados: o primeiro, mais superficial, buscou refinar a escala e identificar um modelo de mensuração adequado ao contexto brasileiro; e o segundo buscou validar nomologicamente a escala purificada na etapa anterior, por meio da incorporação de construtos hipoteticamente antecedentes e consequentes à conveniência.

\section{Estudo 1: identificação de uma escala ampliada}

O Estudo 1 utilizou uma amostra de 268 estudantes universitários (138 mulheres, 127 homens e três que não informaram gênero), com médias de idade de 25,67 e 27,65 anos, respectivamente. A construção de uma escala ampliada, voltada à realidade brasileira, teve como ponto de partida a escala original de Seiders, Voss e Godfrey (2007), que foi sendo adaptada progressivamente, numa abordagem exploratório-confirmatória.

A Análise Fatorial Exploratória de Estrutura Confirmatória (E/CFA), conforme Brown (2006, p. 193), "é utilizada como precursora da CFA, e permite ao pesquisador explorar melhor a estrutura de mensuração antes de passar à abordagem confirmatória”. Segundo esse autor, a E/CFA representa um passo intermediário entre a EFA e a CFA, que oferece algumas informações importantes para a obtenção de uma solução confirmatória realista, as quais nem sempre são obtidas por meio da análise exploratória convencional.

A identificação dos novos construtos foi feita de modo incremental, com a inclusão e teste das novas variáveis uma a uma. Nas diversas estruturas fatoriais que foram exploradas, dois fatores afloraram nitidamente: o primeiro composto por variáveis relacionadas à mobilidade (qualidade do trânsito e dos equipamentos urbanos, existência de tele-entregas e disposição das mercadorias), e o segundo representado por algumas barreiras vivas, como os vendedores ambulantes, os "flanelinhas" e os cães abandonados. Outros possíveis novos fatores também foram investigados, mas foram descartados por instabilidade ou por falta de consistência conceitual. Os dois novos fatores foram analisados quanto à compatibilidade com a pesquisa exploratória e incluídos no modelo confirmatório para verificação da convergência.

A Análise Fatorial Confirmatória ratificou a existência de duas novas dimensões: mobilidade e inconvenientes urbanos. Com exceção da variável "facilidade de encontrar informação sobre a empresa antes de sair às compras", que teve que ser substituída pela "confiança no prestador de serviço", todas as demais variáveis da Servcon foram mantidas no modelo. O estacionamento para clientes, que na escala de Seiders, Voss e Godfrey (2007) aparece como um item da conveniência de acesso, ajustou-se melhor à mobilidade urbana, e ali foi realocado.

O resultado, conforme se vê na Tabela 1 (Estudo 1 ), aponta níveis superiores aos mínimos esperados de confiabilidade $(0,7)$ e de variância extraída $(0,5)$ na maioria dos fatores (BAGOZZI e YI, 1988), e índices aceitáveis de ajustamento geral $(\chi 2=341,3$; G.L. $=252$; $\mathrm{P}=0,0002$; $\mathrm{CFI}=0,917$; TLI=0,902; RMSEA=0,046) (BAUMGARTNER E HOMBURG, 1996).

\section{Estudo 2: confirmação e avaliação da validade nomológica dos novos construtos identificados}

O Estudo 2 ouviu uma amostra de 206 consumidores não acadêmicos, formada por 138 (67\%) mulheres, $63(30,6 \%)$ homens e cinco pessoas que não informaram o gênero, com médias de idade de 30,1 anos (mulheres) e 33,3 anos (homens). Além das variáveis da Escala de Conveniência Ampliada - depurada durante o Estudo 1 - o questionário incluiu questões referentes ao envolvimento com a loja e com os produtos ali vendidos, ao prazer manifesto de comprar, à satisfação com os serviços oferecidos pela loja e à intenção de alguns comportamentos futuros, como a recompra e a recomendação.

O resultado confirmou a existência dos mesmos fatores identificados no Estudo 1, com pequenas diferenças entre as cargas fatoriais. Conforme se vê na Tabela 1, os sete fatores da Escala de Conveniência Ampliada apresentaram níveis de confiabilidade e variância bastante semelhantes em ambas as amostras, e os fatores referentes à satisfação, ao prazer e ao 


\section{Tabela 1 - Modelos de mensuração dos construtos latentes}

\begin{tabular}{|l|c|c|c|c|}
\hline \multirow{2}{*}{ Itens dos modelos de mensuração } & \multicolumn{2}{|c|}{ Estudo 1} & \multicolumn{2}{c|}{ Estudo 2} \\
Cargas & $\begin{array}{c}\text { Confiabilidade } \\
\text { variância }\end{array}$ & $\begin{array}{c}\text { Cargas } \\
\text { padroniz. }\end{array}$ & $\begin{array}{c}\text { Confiabilidade } \\
\text { variância }\end{array}$ \\
\hline
\end{tabular}

1. CONVENIÊNCIA DE DECISÃO

Confiança no prestador de serviço*

\begin{tabular}{l|l|}
\hline 0,814 & 0,597 \\
\hline
\end{tabular}

Decisão fácil*

Conhecimento prévio da empresa*

0,460

0,6571

0,612

$0,711 /$

0,367

0,355

0,47

0,384

Certeza da disponibilidade dos itens de compra**

0,329

0,459

2. CONVENIÊNCIA DE ACESSO

Horários convenientes*

Possivel chegar rapidamente*

Locais convenientes*

\begin{tabular}{|c|c|}
\hline 0,624 & $0,701 /$ \\
0,572 & 0,440 \\
\hline 0,531 & \\
\hline
\end{tabular}

0,459

\section{CONVENIÊNCIA DE TRANSAÇÃO}

Fácil de concluir a transação*

Completar a compra rapidamente*

\begin{tabular}{l|l}
\hline 0,726 & $0,810 /$ \\
0,677 & 0,588 \\
0,630 &
\end{tabular}

Pagar rapidamente*

\section{CONVENIÊNCIA DE BENEFÍCIO}

Fácil conseguir informações sobre os produtos*

Fácil localizar as mercadorias*

Fácil avaliar as mercadorias*

Fácil localizar os produtos*

\section{CONVENIÊNCIA PÓS-BENEFÍCIO}

Fácil efetuar retornos e trocas*

Problemas pós-compra rapidamente resolvidos*

0,674

0,606

$0,801 /$

0,503

0,603

0,595

0,591

0,562

0,495

$0,668 /$

0,402

Troca produtos prontamente*

\begin{tabular}{l|c|}
\hline 0,812 & $0,887 /$ \\
0,658 & 0,725 \\
0,845 &
\end{tabular}

\begin{tabular}{|c|c|}
\hline 0,677 & $0,827 /$ \\
\hline 0,73 & 0,614 \\
\hline 0,68 &
\end{tabular}

\section{INCONVENIENTES URBANOS}

Presença de vendedores ambulantes nas imediações

Presença de cães errantes nas imediações

\begin{tabular}{c|c}
0,818 & $0,905 /$ \\
0,799 & 0,761 \\
0,781 &
\end{tabular}

0,68
0,623
0,64
0,674
0,619

$0,819 /$

0,531

Presença de "guardadores" de carros nas imediações

\section{MOBILIDADE}

Existência de serviços de tele-entrega

Complexidade do trânsito

Disposição da mercadoria e dos equipamentos da Ioja

Qualidade dos equipamentos públicos

0,681

0,834

$0,835 /$

0,685

0,632

0,596

Existência de estacionamento*

0,592
0,534
0,464
0,258

0,318

0,631

$0,641 /$

0,303

0,287

0,645

0,329

$0,775 /$

0,535

0,603

$0,721 /$
0,359

0,287

(continua) 
Tabela 1 - Modelos de mensuração dos construtos latentes

(conclusão)

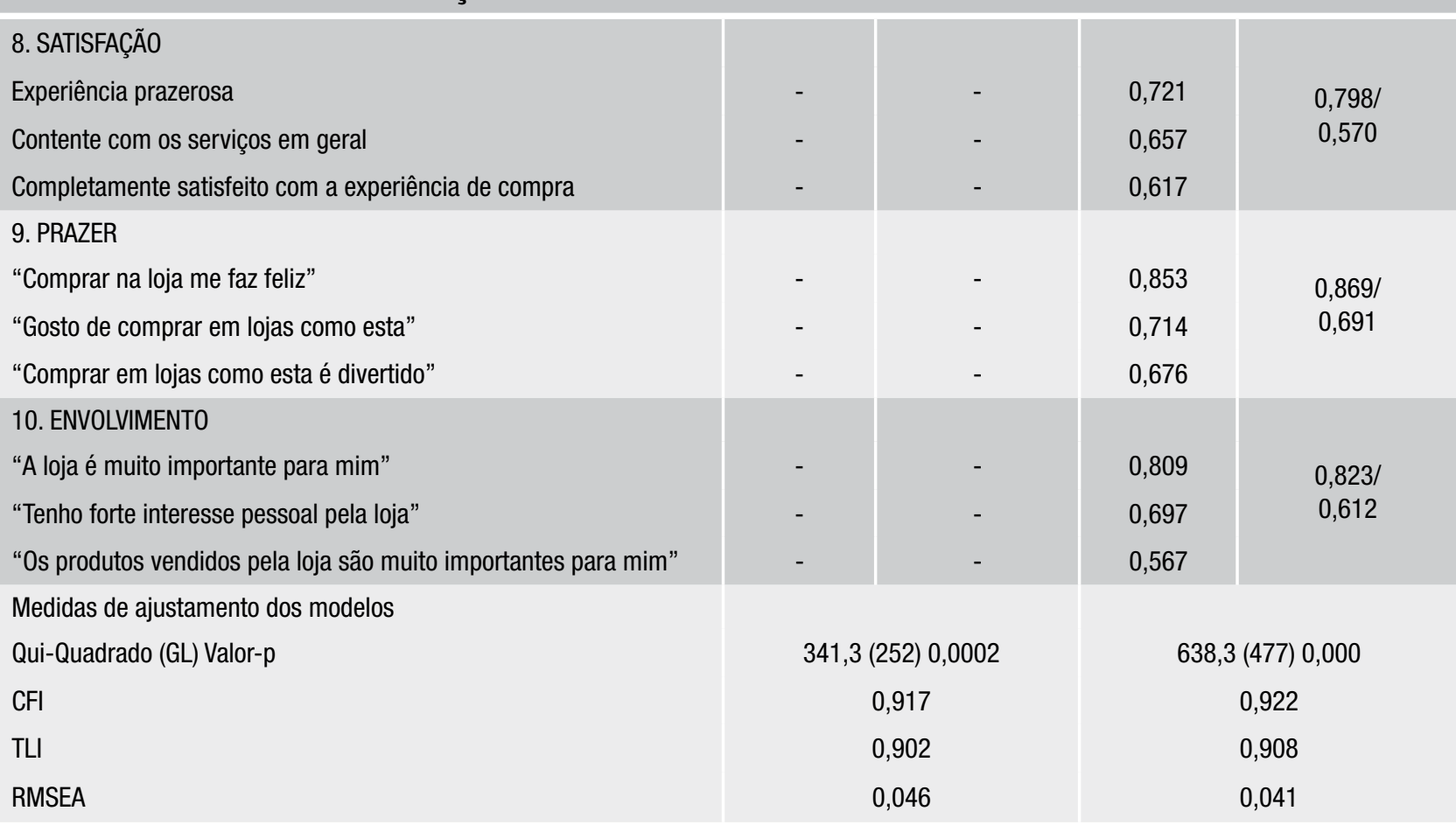

Nota: $\left(^{*}\right)$ variáveis da Servcon original; $\left(^{* *}\right)$ variáveis identificadas durante no trabalho exploratório

envolvimento (Estudo 2) também apresentaram níveis satisfatórios de confiabilidade e de variância extraída.

A validade convergente foi atestada pela significância estatística entre os indicadores e os fatores aos quais estavam relacionados, em ambos os estudos, cujas cargas fatoriais superaram, com folga, o limite mínimo de duas vezes os erros padrões correspondentes (ANDERSON e GERBING, 1988). A validade discriminante foi avaliada pelo método de Fornell e Larcker (1981) (Tabela 2), e os resultados apontaram índices aceitáveis em todos fatores, exceto na conveniência de decisão, cuja correlação com outros fatores supera a variância extraída pelo próprio construto, o que sugere a existência de possível ambiguidade conceitual.

\section{Mensuração dos antecedentes e consequentes}

Além das dimensões da Escala de Conveniência Ampliada, o Estudo 2 mensurou também a satisfação, o prazer de comprar e o envolvimento dos consumidores com os produtos vendidos pelas lojas às quais se referiam. Foram utilizadas as mesmas variáveis empregadas por Seiders, Voss e Godfrey (2007), três para cada construto, cujos indicadores encontram-se demonstrados na Tabela 1. A segurança e a intenção de continuar comprando e de recomendar a loja foram medidas por variáveis individuais, tendo em vista que estas não se agruparam adequadamente em nenhum fator.

\section{As relações estruturais entre os construtos}

As relações de ancestralidade entre os construtos foram testadas por meio de análise de caminhos, com utilização do Software M-Plus (MUTHÉN e MUTHÉN, 1998). O resultado confirma que o envolvimento com os produtos vendidos pela loja tem influência direta na percepção de conveniência de acesso, de transação, de benefício e de pós-benefício, conforme se vê na Tabela 3. Tais constatações ampliam os achados de Seiders, Voss e Godfrey (2007), ao incluir as conveniências de acesso, de transação e de pós-benefício como consequentes do envolvimento, mas não confirmam a relação envolvimento $\rightarrow$ decisão entre os consumidores pesquisados. Mantêm-se, assim, parcialmente a hipótese $H 2$. O resultado também aponta influência direta do prazer de comprar (shopping enjoyment) na satisfação dos consumidores (relação não hipotetizada), mas não se confirma a existência da relação indireta prazer $\rightarrow$ conveniência $\rightarrow$ satisfação, conforme esperado em $H 1$. 


\section{Tabela 2 - Avaliação da validade discriminante}

\begin{tabular}{|l|c|c|c|c|c|c|c|}
\hline Fatores de conveniência & Decisão & Acesso & Transação & $\begin{array}{c}\text { Pés- } \\
\text { Benefício }\end{array}$ & $\begin{array}{c}\text { Inconvenientes } \\
\text { uenefício }\end{array}$ & $\begin{array}{c}\text { Mrbanosilidade } \\
\text { urbanos }\end{array}$ \\
\hline Conv. Decisão & 1 & 0,80 & 0,89 & 0,99 & 0,63 & 0,16 \\
\hline Conv. Acesso & 0,65 & 1 & 0,54 & 0,54 & 0,38 & 0,16 \\
\hline Conv. Transação & 0,79 & 0,29 & 1 & 0,72 & 0,51 & 0,13 & 0,21 \\
\hline Conv. Benefício & 0,97 & 0,29 & 0,51 & 1 & 0,55 & 0,12 & 0,27 \\
\hline Conv. Pós-Benefício & 0,40 & 0,15 & 0,26 & 0,31 & 1 & 0,23 & 0,21 \\
\hline Inconvenientes Urbanos & 0,03 & 0,02 & 0,02 & 0,01 & 0,05 & 0,79 \\
\hline Mobilidade & 0,11 & 0,05 & 0,02 & 0,07 & 0,04 & 0,63 \\
\hline Confiabilidade Composta & 0,711 & 0,668 & 0,827 & 0,819 & 0,835 & 0,775 \\
\hline Variância Extraída & 0,384 & 0,402 & 0,614 & 0,531 & 0,632 & 0,535 \\
\hline
\end{tabular}

Nota: os valores acima da diagonal principal correspondem às correlações entre os construtos, e os valores opostos correspondem às correlações elevadas ao quadrado

A percepção de segurança contribui diretamente para a decisão de compra - e, consequentemente, na escolha do fornecedor - conforme hipotetizado (H3), mas não chega a influenciar diretamente a satisfação, a decisão de recomprar ou de recomendar.

Conforme hipotetizado, esperava-se que as lojas localizadas em áreas de grande concentração comercial e administrativa apresentassem maior restrição à mobilidade, mas que, mesmo assim, fossem vistas como mais convenientes, devido à convergência das vias e dos meios de transporte e à complementaridade (GIJSBRECHTS, CAMPO, NISOL, 2008). O resultado confirma que o acesso às lojas localizadas no Centro, especialmente aquelas localizadas no Calçadão de Pelotas/RS (área destinada à circulação exclusiva de pedestres) e na Zona Norte, é visto de modo significativamente mais conveniente do que nas demais localizações $(H 4 b)$. Confirma-se também a percepção de maior mobilidade, entre os clientes das lojas localizadas em áreas de comércio emergente, como na Zona Norte e no Areal ( $H 4 a)$, e a influência negativa dos chamados inconvenientes urbanos (informalidade, desorganização e mendicância), nas lojas localizadas no Centro e no Calçadão ( $H 4 C$ ).

Não se confirma a existência de relação direta entre a mobilidade e a satisfação, a recomendação e recompra, conforme hipotetizado em $H$ - 6 . Entretanto, confirma-se a influência direta e significativa da conveniência de benefício na satisfação $(H 7)$, da satisfação nas decisões de falar positivamente e de recomendar os serviços da loja (H8).
Confirma-se a influência da conveniência de acesso e de benefício na de decisão, mas não há evidências significativas de que a facilidade de transação ou de obtenção de benefícios posteriores funcionem do mesmo modo. Mantém-se, assim, parcialmente a hipótese $H 9$, com também se mantém a hipótese $H 10$, de que a conveniência de benefício é positivamente influenciada pela conveniência de transação.

O resultado confirma que a presença de informais, de animais errantes e de suplicantes em áreas de grande fluxo de público reduz significativamente a mobilidade e a sensação de segurança dos consumidores (uma relação não hipotetizada), e causa desconforto aos consumidores (H5), especialmente às mulheres. Entretanto, não se confirma a existência de diferenças significativas entre as diversas faixas de idade, no que tange ao desconforto provocado por tais inconvenientes. A Tabela 4 apresenta as relações entre os chamados inconvenientes urbanos e o gênero e a percepção de segurança das pessoas.

Além das relações hipotetizadas, também foram encontradas algumas relações não hipotetizadas que poderão ser úteis para fins gerenciais. É o caso da influência da conveniência de acesso, de benefício e de pós-benefício, no comportamento de recompra, a qual não deveria ocorrer de maneira direta, mas sim por meio da satisfação. E também da significância encontrada entre a idade dos consumidores e a as lojas localizadas no Bairro Areal, cuja presença pode estar associada à existência de algumas lojas mais seletivas naquela área. A Tabela 5 resume os resultados das 


\section{Tabela 3 - Relações estruturais entre os fatores de conveniência e seus antecedentes e consequentes (variáveis dependentes intervalares)}

\begin{tabular}{|c|c|c|c|c|c|c|}
\hline Construtos antecedentes & & $\begin{array}{l}\text { Construtos } \\
\text { consequentes }\end{array}$ & Cargas padroniz. & E.P. & $\begin{array}{c}\text { Carga } \\
\text { /E.P. }\end{array}$ & Sig. \\
\hline Conv. acesso & $\rightarrow$ & Conv. decisão & 0,380 & 0,072 & 5,28 & 0,000 \\
\hline Conv. acesso & $\rightarrow$ & Recompra & 0,308 & 0,068 & 4,53 & 0,000 \\
\hline Conv. benefício & $\rightarrow$ & Conv. decisão & 0,933 & 0,062 & 15,05 & 0,000 \\
\hline Conv. benefício & $\rightarrow$ & Satisfação & 0,330 & 0,073 & 4,52 & 0,000 \\
\hline Conv. benefício & $\rightarrow$ & Recompra & 0,286 & 0,061 & 4,69 & 0,000 \\
\hline Conv. pós-benefício & $\rightarrow$ & Recompra & 0,180 & 0,057 & 3,16 & 0,002 \\
\hline Conv. transação & $\rightarrow$ & Conv. benefício & 0,538 & 0,072 & 7,47 & 0,000 \\
\hline Envolvimento & $\rightarrow$ & Conv. benefício & 0,385 & 0,075 & 5,13 & 0,000 \\
\hline Envolvimento & $\rightarrow$ & Conv. acesso & 0,551 & 0,068 & 8,10 & 0,000 \\
\hline Envolvimento & $\rightarrow$ & Transação & 0,647 & 0,048 & 13,48 & 0,000 \\
\hline Envolvimento & $\rightarrow$ & Pós-benefício & 0,565 & 0,057 & 9,91 & 0,000 \\
\hline Inconv. urbanos & $\rightarrow$ & Mobilidade & 0,857 & 0,092 & 9,32 & 0,000 \\
\hline Localiz. calçadão & $\rightarrow$ & Inconv. urbanos & $(0,176)$ & 0,082 & $(2,15)$ & 0,031 \\
\hline Localiz. calçadão & $\rightarrow$ & Conv. acesso & 0,400 & 0,152 & 2,63 & 0,008 \\
\hline Localiz. areal & $\rightarrow$ & Mobilidade & 0,506 & 0,087 & 5,82 & 0,000 \\
\hline Localiz. areal & $\rightarrow$ & Conv. acesso & 0,223 & 0,078 & 2,86 & 0,004 \\
\hline Localiz. Centro & $\rightarrow$ & Inconv. urbanos & $(0,181)$ & 0,085 & $(2,13)$ & 0,033 \\
\hline Localiz. Centro & $\rightarrow$ & Conv. acesso & 0,215 & 0,134 & 1,60 & 0,108 \\
\hline Localiz. Zona Norte & $\rightarrow$ & Mobilidade & 0,516 & 0,093 & 5,55 & 0,000 \\
\hline Localiz. Zona Norte & $\rightarrow$ & Conv. acesso & 0,207 & 0,090 & 2,30 & 0,022 \\
\hline Prazer & $\rightarrow$ & Satisfação & 0,691 & 0,067 & 10,31 & 0,000 \\
\hline Satisfação & $\rightarrow$ & Falar positivamente & 0,627 & 0,041 & 15,29 & 0,000 \\
\hline Satisfação & $\rightarrow$ & Recomendar & 0,666 & 0,042 & 15,86 & 0,000 \\
\hline Segurança & $\rightarrow$ & Decisão & 0,107 & 0,045 & 2,38 & 0,018 \\
\hline
\end{tabular}

Nota: estimador $=$ MLM; $\chi 2=1140 ; \mathrm{GL}=766 ; \mathrm{Sig}=0,000 ; \mathrm{CFI}=0,865 ; \mathrm{TLI}=0,850 ; \mathrm{RMSEA}=0,050$

Tabela 4 - Relações estruturais entre os fatores de conveniência e seus consequentes (variáveis dependentes categóricas)

\begin{tabular}{|l|l|l|c|c|c|c|}
\hline $\begin{array}{c}\text { Construto/Variável } \\
\text { antecedente }\end{array}$ & & & $\begin{array}{c}\text { Cargas } \\
\text { padronizadas }\end{array}$ & E.P. & $\begin{array}{c}\text { Carga } \\
\text { /E.P. }\end{array}$ & Sig. \\
\hline Gênero (f) & $\rightarrow$ & Inconvenientes urbanos & $-0,483$ & 0,236 & $-2,047$ & 0,041 \\
\hline Inconvenientes urbanos & $\rightarrow$ & Segurança & 0,283 & 0,066 & 4,288 & 0,000 \\
\hline
\end{tabular}

Nota: estimador $=$ WLSMV; $\chi 2=25,5 ; \mathrm{GL}=15 ; \mathrm{Sig}=0,043 ; \mathrm{CFI}=0,915 ; \mathrm{TLI}=0,915 ; \mathrm{RMSEA}=0,059$ 
relações hipotetizadas, assim como alguns achados inesperados que poderão ser úteis em trabalhos futuros. É o caso, por exemplo, das relações diretas [prazer (shopping enjoyment) $\rightarrow$ satisfação] e [informalidade $\rightarrow$ segurançal, cujo conhecimento ainda comporta alguns avanços.

\section{DISCUSSÃO}

A pesquisa parte do pressuposto de que a Servcon, desenvolvida por Seiders, Voss e Godrey (2007) com o propósito de medir a conveniência dos serviços, não é abrangente o suficiente para avaliar aquele construto na complexa realidade urbana brasileira. Ainda que a escala original tenha se mostrado válida e confiável no contexto onde foi desenvolvida, as suas cinco dimensões não chegam a considerar algumas causas de desconforto que são típicas das cidades brasileiras, mormente das cidades de médio porte, como o trânsito, a insegurança e a informalidade.

O trabalho inova sobre a escala original, na medida em que incorpora alguns elementos de desconforto que são típicos de países em desenvolvimento, mas que não haviam sido considerados por ocasião da criação da Servcon. É o caso, por exemplo, da conveniência de acesso, definida por Berry, Seiders e

\section{Tabela 5 - Resultado resumido das hipóteses testadas}

\begin{tabular}{|c|c|c|c|c|}
\hline Hipótese & \multicolumn{3}{|c|}{ Relação esperada } & Resultado \\
\hline $\mathrm{H} 1$ & Prazer de comprar & $\begin{array}{l}\rightarrow \\
\#\end{array}$ & $\begin{array}{l}\text { Conveniência (5 dimensões) } \\
\text { Satisfação }\end{array}$ & $\begin{array}{l}\text { Ausente } \\
\text { Presente }\end{array}$ \\
\hline $\mathrm{H} 2$ & Envolvimento & $\begin{array}{l}\rightarrow \\
\rightarrow \\
\#\end{array}$ & $\begin{array}{l}\text { Conveniência de decisão } \\
\text { Conveniência de benefício } \\
\text { Conveniência de acesso, de transação e de } \\
\text { pós-benefício }\end{array}$ & $\begin{array}{l}\text { Ausente } \\
\text { Mantida } \\
\text { Presente }\end{array}$ \\
\hline $\mathrm{H} 3$ & Segurança & $\begin{array}{l}\rightarrow \\
\rightarrow\end{array}$ & $\begin{array}{l}\text { Conveniência de decisão } \\
\text { Satisfação, recompra e recomendação }\end{array}$ & $\begin{array}{l}\text { Mantida } \\
\text { Ausente }\end{array}$ \\
\hline $\mathrm{H} 4 \mathrm{a}$ & Aglomeração & $\rightarrow$ & Redução da mobilidade & Mantida \\
\hline $\mathrm{H} 4 \mathrm{~b}$ & Aglomeração & $\rightarrow$ & Conveniência de acesso & Mantida \\
\hline $\mathrm{H} 4 \mathrm{C}$ & Aglomeração & $\rightarrow$ & $\begin{array}{l}\text { Maior desconforto com os inconvenientes } \\
\text { urbanos }\end{array}$ & Mantida \\
\hline H5 & Informalidade & $\begin{array}{l}\rightarrow \\
\rightarrow \\
\rightarrow \\
\#\end{array}$ & $\begin{array}{l}\text { Redução da mobilidade } \\
\text { Desconforto maior entre as mulheres } \\
\text { Desconforto maior entre os mais velhos } \\
\text { Segurança }\end{array}$ & $\begin{array}{l}\text { Mantida } \\
\text { Mantida } \\
\text { Ausente } \\
\text { Presente }\end{array}$ \\
\hline $\mathrm{H} 6$ & Mobilidade & $\rightarrow$ & Satisfação, recomendação e recompra & Ausente \\
\hline $\mathrm{H} 7$ & $\begin{array}{l}\text { Conveniência de benefício } \\
\text { Conveniência de acesso, de decisão, de tran- } \\
\text { sação e de pós-benefício }\end{array}$ & $\begin{array}{l}\rightarrow \\
\rightarrow\end{array}$ & $\begin{array}{l}\text { Satisfação } \\
\text { Satisfação }\end{array}$ & $\begin{array}{l}\text { Mantida } \\
\text { Ausente }\end{array}$ \\
\hline $\mathrm{H} 8$ & Satisfação & $\rightarrow$ & Recompra e recomendação & Mantida \\
\hline $\mathrm{H} 9$ & $\begin{array}{l}\text { Conveniência de acesso e de benefício } \\
\text { Conveniência de transação e de pós-benefício }\end{array}$ & $\begin{array}{l}\rightarrow \\
\rightarrow\end{array}$ & $\begin{array}{l}\text { Conveniência de decisão } \\
\text { Conveniência de decisão }\end{array}$ & $\begin{array}{l}\text { Mantida } \\
\text { Ausente }\end{array}$ \\
\hline $\mathrm{H} 10$ & Conveniência de transação & $\rightarrow$ & Conveniência de benefício & Mantida \\
\hline
\end{tabular}

Nota: 0 símbolo "\#” corresponde a achados espúrios (relações não hipotetizadas) 
Grewal (2002) como o tempo e o esforço gastos para se ter acesso ao serviço, que não leva em consideração a estrutura urbana, a complexidade do sistema viário e as barreiras físicas e humanas que são próprias da informalidade terceiro-mundista.

A conveniência de acesso, na forma como foi concebida originalmente, assume que o deslocamento dos consumidores até a loja (quando necessário) se dá exclusivamente de carro, o que não corresponde à realidade de boa parte dos brasileiros. Ao deixar de fora algumas questões como a disponibilidade, a qualidade e a segurança do transporte coletivo, os trabalhos anteriores ignoraram alguns elementos de extrema importância para os consumidores brasileiros, em especial para aqueles que residem na periferia das cidades.

A conveniência de decisão passou por algumas adequações, mas ainda se mostra frágil do ponto de vista conceitual e estatístico. Conforme Berry, Seiders e Grewal (2002, p. 6), "a decisão de compra envolve decisões sobre o tipo de produto a comprar e o fornecedor a ser escolhido". E a conveniência de decisão corresponde "ao tempo e ao esforço percebidos pelo consumidor, para fazer as escolhas de compra". Na visão daqueles autores, ao que parece, a conveniência de decisão seria formada por componentes preponderantemente objetivos (facilidade de encontrar informações, certeza de encontrar o que se busca e decisão fácil e rápida), mas não contemplaria aspectos afetivos, como alguns que foram identificados no presente trabalho (confiança e relacionamento prévio com a loja).

A inadequação da variável original "abundância de informações sobre a loja" e a identificação de duas outras associadas à redução da incerteza (confiança no prestador de serviço e conhecimento prévio da empresa) sugerem que, além do tempo e do esforço para decidir, a conveniência de decisão também contempla aspectos do afeto e do hábito, que podem ser tão importantes quanto a facilidade de encontrar informações sobre o prestador de serviços.

O presente trabalho apresenta evidências de que a conveniência de decisão, da forma como foi concebida originalmente, não se ajusta à realidade brasileira, mas não logra esgotar o assunto. A inexistência de relação significante entre o envolvimento e essa dimensão da conveniência, além do baixo índice de variância extraída pelo fator e dos elevados coeficientes de correlação com outros fatores, reduz a validade discriminante e sugere a necessidade de reespecificar novamente $\mathrm{O}$ construto. Também a constatação de que a segurança influi direta e significativamente na conveniência de decisão, sem fazer parte desta, sugere a necessidade de alargamento do espectro de variáveis referentes ao risco físico, financeiro ou psicológico, em trabalhos futuros, para confirmar se a segurança, efetivamente, constitui um construto à parte ou se, eventualmente, pode ser incorporada à conveniência de decisão ou a outro fator.

Nem todas as relações hipotetizadas se confirmaram, e outras não esperadas foram identificadas. É o caso, por exemplo, das relações entre o "prazer de ir às compras" (shopping enjoyment) e os diversos fatores de conveniência, que não se confirmam da forma como foram hipotetizadas (prazer $\rightarrow$ conveniência $\rightarrow$ satisfação), mas que aparecem diretamente como prazer $\rightarrow$ satisfação; e do desconforto causado pela informalidade, que se confirma apenas em relação ao gênero (é maior entre as mulheres), mas que não se confirma em relação aos mais velhos. A ausência de tais relações possivelmente se deva às características da amostra, formada preponderantemente por pessoas jovens, que se locomovem com maior facilidade, mesmo em áreas obstaculizadas.

O trabalho inova na identificação de relações significativas entre alguns construtos da própria conveniência, como é o caso da conveniência de decisão, que depende da facilidade de acesso e dos benefícios; e da própria conveniência de benefício, que depende da rapidez e da eficiência da transação. Inova, também, na identificação de relação direta entre a conveniência de pós-benefício e a decisão de recompra, independentemente da satisfação, o que pode ser de grande interesse para os estudos evolvendo a conquista e a manutenção da lealdade. E inova, fundamentalmente, ao confirmar que a percepção de segurança influencia significativamente a conveniência de decisão.

A pesquisa avança na identificação de novas dimensões de conveniência, em especial dos inconvenientes urbanos, que apresentam bons índices de confiabilidade, de validade e de variância extraída, porém sem esgotar o assunto. A constatação de que os inconvenientes urbanos interferem na mobilidade das pessoas e de que esta varia significativamente de um local para outro, conforme a densidade comercial e o gênero dos respondentes, sugere que esses fatores merecem ser incorporados às estratégias do varejo. A não confirmação de relações diretas entre a mobilidade e a satisfação dos clientes (HO) é intrigante e também merece ser mais bem investigada. E o mesmo pode ser dito em relação à segurança, cuja influência sobre a conveniência de decisão abre uma nova senda de pesquisa sobre o assunto, mas que 
precisa ser ampliada enquanto construto, tendo em vista que a sua operacionalização restringiu-se a uma única variável métrica.

O trabalho tem limitações quanto ao tamanho da amostra, em especial a amostra que foi empregada no estudo de validação (Estudo 2), e quanto à ausência de um conjunto mais abrangente de variáveis referentes à segurança. A sensação de segurança, avaliada da forma como foi (por meio de uma única variável), é conceitualmente imprecisa. Um respondente pode se dizer inseguro por ter sido assaltado numa de suas idas ao comércio (falta de segurança pública), ou por não ver um grande número de policiais nas ruas; mas pode, também, se sentir inseguro pelo contrário: por ter que conviver com um aparato preventivo-ostensivo e indesejável (carros-fortes, guardas armados, portas giratórias, câmeras etc.). Por essa razão, sugere-se que trabalhos futuros contemplem um elenco mais generoso de variáveis representativas da (in)segurança.

O presente trabalho tem implicações gerenciais para os gestores de marketing, seja para o posicionamento ou reposicionamento de serviços, seja para definir estratégias de distribuição ou de instalação de novos pontos de venda. E poderá ser particularmente útil para os gestores públicos encarregados do planejamento da segurança ou do regramento das atividades informais e da mobilidade urbana, que poderão se valer da Escala Ampliada para medir o impacto dos obstáculos vivos e da mobilidade na percepção de conveniência nos diversos pontos e vias das médias e grandes cidades.

\section{NOTA DE AGRADECIMENTO}

Os autores agradecem ao CNPQ pelo apoio financeiro recebido para realização da pesquisa.

\section{REFERÊNCIAS}

ALCÂNTARA, P. I. Corporação de informais. Desafios do Desenvolvimento IPE, n. 9, 2005. Disponível em: http:// www.ipea.gov.br/desafios/edicoes/9/sumario.php. Acesso em 09.02.2009.

AMIGOS DE PELOTAS. Cachorro, um ser onipresente em Pelotas. Blog Amigos de Pelotas, 2008. Disponível em: http://www.amigosdepelotas.com/2008/11/cachorro-um-ser-onipresente-em-pelotas.html. Acesso em 18.07.2011.
ANDERSON, E. W. Customer satisfaction and word of mouth. Journal of Service Research, v. 1, n. 1, p. 5-17, 1998.

ANDERSON, J. C; GERBING, D. W. Structural equation modeling in practice: a review and recommended two-step approach. Psychological Bulletin, v. 103, n. 3, p. 411-423, 1988.

ARNOLD, M. J; REYNOLDS, K. E. Hedonic shopping motivations. Journal of Retailing, v. 79, n. 2, p. 77-95, 2003.

BAGOZZI, R. P; Yi, Y. On the evaluation of structural equation models. Journal of the Academy of Marketing Science, v. 16, n. 1, p. 74-94, 1988.

BAKER, J; PARASURAMAN, A; GREWAL, D; VOSS, G. B. The influence of multiple store environment cues on perceived merchandise value and patronage intentions. Journal of Marketing, v. 66, n. 2, p. 120-141, 2002.

BAUMGARTNER, H; HOMBURG, C. Applications of structural equation modeling in marketing and consumer research: a review. International Journal of Research in Marketing, v. 13, n. 2, p. 139-161, 1996

BEATTY, S. E; SMITH, S. M. External search effort: an investigation across several product categories. Journal of Consumer Research, v. 14, n. 1, p. 83-95, 1987.

BERRY, L. L; SEIDERS, K; GREWAL, D. Understanding service convenience. Journal of Marketing, v. 66, n. 3, p. 1-17, 2002.

BROWN, L. G. Convenience in services marketing. Journal of Services Marketing, v. 4, n. 1, p. 53-59, 1990.

BROWN, L. G; MCENALLY, M. R. Convenience: definition, structure, and application. The Journal of Marketing $\mathrm{Ma}$ nagement, v. 2, n. 2, p. 47-56, 1993.

BROWN, T. A. Confirmatory factor analysis for applied research. New York: The Guilford Press, 2006.

CHESNAIS, J. C. A violência no Brasil: causas e recomendações políticas para a sua prevenção. Ciência \& Saúde Coletiva, v. 4, n. 1, p. 53-69, 1999. 
COLLIER, J. E; SHERRELL, D. L. Examining the influence of control and convenience in a self-service setting. Journal of the Academy of Marketing Science, v. 38, n. 4, p. 490-509, 2010.

COLWELl, S. R; AUNG, M; KANETKAR, V; HOLDEN, A. L. Toward a measure of service convenience: multiple-item scale development and empirical test. Journal of Service Marketing, v. 22, n. 2, p. 160-169, 2008.

DABHOLKAR, P. A; THORPE, D. I; RENTZ, J. O. A measure of service quality for retail stores: scale development and validation. Journal of the Academy of Marketing Science, v. 24, n. 1, p. 3-16, 1996.

FERREIRA, D. F. Belém é invadida por flanelinhas. Jornal O Liberal, 03.06.2006. Disponível em: http://www.soleis. adv.br/artigoflanelinhas.htm. Acesso em 09.02.2009.

FORNEL, C; LARCKER, D. F. Evaluating structural equation models with unobservable variables and measurement errors. Journal of Marketing Research, v. 18, n. 1, p. 39-50, 1981.

GIJSBRECHTS, E; CAMPO, K; NISOL, P. Beyond promotion-based store switching: Antecedents and patterns of systematic multiple-store shopping. International Journal of Research in Marketing, v. 25, n. 1, p. 5-21, 2008.

GIL, W. Excesso de ambulantes prejudica circulação no centro da cidade. Jornal Vanguarda. Caruaru, 2011. Disponível em: http://www.jornalvanguarda.com.br/ v2/?pagina=noticias\&id=8605. Acesso em 18.07.2011.

GOSS, J. The "magic of the mall": An analysis of form, function, and meaning in the contemporary retail built environment. Annals of the Association of American Geographers, v. 83, n. 1, p. 18-47, 1993. Disponível em: http://www.jstor. org/stable/2569414. Acesso em 02.08.2010. Disponível em: http://www.jstor.org/stable/2569414

MELLO, D. A favelização de pelotas. Diamond City, 2011. Disponível em: http://ladointerativo. net/david/post2. php?id=325. Acesso em 18.07.2011.

MEUTER, M. L; OSTROM, A. L; ROUNDTREE, R. I; BITNER, M. J. Self-service technologies: understanding customer satisfaction with technology-based service encounters. Journal of Marketing, v. 64, n. 3, p. 50-64, 2000.
MILLER, C. E; REARDON, J; MCCORKLE, D. E. The effects of competition on retail structure: an examination of intratype, intertype, and intercategory competition. Journal of Marketing, v. 63, n. 4, p. 107-120, 1999.

MUTHÉN, L. K; MUTHÉN, B. O. MPlus: statistical analysis with latent variables - user's guide. Los Angeles: Muthén \& Muthén, 1998.

SANTOS, C. P; FERNANDES, D. V. H. A recuperação de serviços como ferramenta de relacionamento e seu impacto na confiança e lealdade dos clientes. RAE-Revista de Administração de Empresa, v. 48, n. 1, p. 10-24, 2008.

SANTOS, C. P; ROSSI, C. A. V. O impacto do gerenciamento de reclamações na confiança e na lealdade do consumidor. Revista de Administração Contemporânea, v. 6, n. 2, p. 49-73, 2002.

SEIDERS, K; BERRY, L. L; GRESHAM, L. Attention retailers: how convenient is your convenience strategy? Sloan Management Review, v. 49, n. 3, p. 79-90, 2000.

SEIDERS, K; VOSS, G. B; GODFREY, A. L. Do satisfied consumers buy more? Examining moderating influences in a retailing context. Journal of Marketing, v. 69, n. 4, p. 26-43, 2005.

SEIDERS, K; VOSS, G. B; GODFREY, A. L. SERVCON: development and validation of a multidimensional service convenience scale. Journal of the Academy of Marketing Science, v. 35, n. 1, p. 144-156, 2007.

SCOTTI, M. Professor do ISAE analisa boom de shopping centers. ISAE-FGV, 2007. Disponível em: http://www.isaebrasil.com.br/novo3/artigos.asp?IdArtigo=15\&origem=home. Acesso em 8.2.2010.

YAMAWAKI, Y; DUARTE, F. Shopping centers e recuperação urbana: estudo empírico de uma contradição teórica. REDES, v. 15, n. 1, p. 32-51, 2010.

ZEITHAML, V. A; BERRY, L. L; PARASURAMAN, A. The behavioral consequences of service quality. Journal of Marketing, v. 60, n. 2, p. 31-46, 1996.

ZEITHAML, V. A. Consumer perceptions of price, quality, and value: a means-end model and synthesis of evidence. Journal of marketing, v. 52, n. 2, p. 2-22, 1988. 